\title{
Estrogen suppresses epileptiform activity by enhancing Kv4.2-mediated transient outward potassium currents in primary hippocampal neurons
}

\author{
YUWEN ZHANG* ${ }^{*}$ YIAN HUANG ${ }^{*}$, XU LIU* ${ }^{*}$ GUOXIANG WANG, XIN WANG and YUN WANG \\ Department of Neurology, Zhongshan Hospital, Collaborative Innovation Center for Brain Science, Institutes of Brain Science \\ and State Key Laboratory for Medical Neurobiology, Fudan University, Shanghai 200032, P.R. China
}

Received February 12, 2015; Accepted July 8, 2015

DOI: $10.3892 / \mathrm{ijmm} .2015 .2287$

\begin{abstract}
Catamenial epilepsy is a common phenomenon in female epileptic patients that is, in part, influenced by the 17- $\beta$-estradiol level during the menstrual cycle, which modulates the strength of the epileptic seizures. However, the underlying mechanism(s) for catamenial epilepsy remains unknown. In the present study, the effect of $17-\beta$-estradiol on modulating epileptiform activities was investigated in cultured hippocampal neurons by focusing on the transient outward potassium current. Using the patch clamp technique, $17-\beta$-estradiol was demonstrated to have a dose-dependent U-shape effect on epileptiform bursting activities in cultured hippocampal neurons; only the low dose $(\sim 0.1 \mathrm{ng} / \mathrm{ml})$ of $17-\beta$-estradiol had a suppressive effect on the epileptiform activities. The blockade effect of the low dose 17- $\beta$-estradiol could be suppressed by phrixotoxin2 (PaTx2), a selective channel blocker for voltage-gated potassium channel type 4.2 (Kv4.2), which mediates the transient outward potassium current. Furthermore, the 17- $\beta$-estradiol bell-shape-like dose-dependently enhanced the transient outward potassium current, which was inhibited by the estrogen receptor antagonist ICI 182,780 . In conclusion, these results indicate that reduced activation of the transient outward potassium current by a high (or none) 17- $\beta$-estradiol level may enhance the epileptiform bursting activities in neurons, which may be one of the triggering causes for catamenial epilepsy, and therefore, maintaining a certain low $17-\beta$-estradiol level may aid in the control of catamenial epilepsy.
\end{abstract}

Correspondence to: Dr Yun Wang or Dr Xin Wang, Department of Neurology, Zhongshan Hospital, Collaborative Innovation Center for Brain Science, Institutes of Brain Science and State Key Laboratory for Medical Neurobiology, Fudan University, Shanghai 200032, P.R. China

E-mail: yunwang@fudan.edu.cn

E-mail: wang.xin@zs-hospital.sh.cn

${ }^{*}$ Contributed equally

Key words: potassium channel, Kv4.2, estrogen receptor, epilepsy

\section{Introduction}

Epilepsy is one of the most common diseases of the central nervous system. In certain female patients, seizures may cluster in association with the menstrual cycle, and this is known as catamenial epilepsy (1). The association between seizures and fluctuations of the sex hormone levels during the ovarian cycles in certain women with epilepsy was suggested as early as the 19th century (2). Since then, clinicians have investigated this observation further and identified that it may be attributed to the neuroactive properties of steroid hormones and their cyclic variation in serum levels. However, the association between estrogens and epileptic seizures remains unclear (3).

Voltage-gated potassium (Kv) channels are important physiological regulators of membrane potentials, action potential shape, firing adaptation and neuronal excitability in excitable tissues (4). The voltage-gated potassium (Kv) currents are divided into two sections: The transient current and the sustained current $(5,6)$. Previous studies have demonstrated that the transient outward potassium current, also known as the A-type potassium current $\left(\mathrm{I}_{\mathrm{A}}\right)$, has an important role in controlling the membrane excitability and it contributes to remodel neuronal excitation under pathological conditions $(7,8)$. In the $\mathrm{Kv}$ channel family, Kv4 is the major subtype mediating the A-type current $\left(\mathrm{I}_{\mathrm{A}}\right) . \mathrm{I}_{\mathrm{A}}$ is encoded by homomultimeric or heteromultimeric complexes of the $\mathrm{Kv}$ channel subunits within identical subfamilies $(9,10)$. In the hippocampus, dendrites of CA1 pyramidal neurons contain a high density of transient A-type potassium channels, and this dampening effect reduces the ability of dendrites to initiate action potentials, decreases the amplitude of back-propagating action potentials, and reduces the magnitude of EPSPs (11). Taken together, these reveal that the A-type potassium current has an inhibitory modulation effect on neuronal activities in the hippocampus, which suggest that inhibition of $\mathrm{Kv}$ channels would increase the cell excitability. Indeed, in pilocarpine seizure rat model, by using electrophysiological recording, the function of A-type potassium channels were found to be decreased, which most likely accounts for the increased dendritic excitability and the epileptic seizure generation (12).

In one of our previous studies, we reported that phenol red, a weak estrogen receptor agonist, as well as $17-\beta$-estradiol, by 
activation of estrogen receptors, exhibited a dose-dependent U-shape-like effect on the spontaneous epileptiform bursting activities in cultured hippocampal neurons (13); however, the underlying mechanism remains unknown. Hoffman et al (14) also reported that modulation of voltage-gated potassium channels by estrogen reduced seizure and induced the spread and degree of neuronal loss in the kainate acid (KA) epilepsy model, which was modulated by progesterone (15). Previous studies have shown that estrogen receptor activation affects the function of various potassium channels in the brain. $17-\beta$-estradiol has been reported to inhibit the activity of the outward potassium currents in the rat parabrachial nucleus (16) and modulate the small conductance calcium-activated SK3 potassium channels in the hypothalamus of the female guinea pig (17). In GT1-7 cells, estrogen has been reported to enhance the Kv4.2 mRNA expression level and transient outward current (18). Thus, activation of estrogen receptors is likely to affect the neuronal excitability and in turn reduces the occurrence of epilepsy, and potassium channels may be involved in this process. However, the mechanism of how estrogen receptor activation modulates the transient outward potassium channel and affects the epileptiform activities is not fully understood.

Therefore, we hypothesized that estrogen modulation of epileptiform activity may occur, at least in part, via affecting the transient outward potassium current. In the present study, the effect of estrogen on the excitability and outward potassium currents of cultured hippocampal neurons was investigated. The results showed that $17-\beta$-estradiol has a suppressive effect on the epileptiform bursting activities in cultured hippocampal neurons and an enhancing effect on the transient outward potassium currents with the similar dose-related U-shape manner. Inhibition of the transient outward potassium currents reversed the low dose 17- $\beta$-estradiol-evoked inhibition on epileptiform bursting activities, indicating that the transient outward potassium currents mediated the anti-seizure effect of low dose 17- $\beta$-estradiol.

\section{Materials and methods}

Ethics statement. All the animal experiments were approved by the Local Committees of the Use of the Laboratory Animals, Fudan University (Shanghai, China) and were carried out in accordance with the National Natural Science Foundation of China animal research regulation.

Primary hippocampal neuronal culture. Primary hippocampal neurons were prepared from embryonic day 18 Sprague-Dawley rats, as previously reported (19). Briefly, the pregnant rat was anaesthetized with $10 \%$ chloral hydrate (intraperitoneal injection), and the pups were dissected out for tissue preparation. All the animals were subsequently euthanized with an overdose of chloral hydrate. Following the dissection of the hippocampus, the tissue was rinsed in cold Hanks' balanced salt solution and subsequently digested with $0.05 \%$ trypsin-ethylenediaminetetraacetic acid for $\sim 15 \mathrm{~min}$ at $37^{\circ} \mathrm{C}$, followed by trituration with pipettes in the plating media (Dulbecco's modified Eagle's medium with $10 \%$ fetal bovine serum, $10 \% \mathrm{~F} 12$ and $25 \mu \mathrm{g} / \mathrm{ml}$ penicillin/streptomycin). Subsequent to rinsing twice, cells were counted and plated onto glass coverslips (12-mm round; Carolina Biological Supply
Co., Burlington, NC, USA) or 35-mm petri dishes with a 20-mm glass bottom well (Shengyou Biotechnology Co., Ltd., Hangzhou, China) precoated with $0.1 \mathrm{mg} / \mathrm{ml}$ poly-D-lysine (Sigma Aldrich, St. Louis, MO, USA). Subsequent to culturing for 1 day, half of the media was changed into neuronal culture media (neurobasal media containing 2 mM GlutaMAX ${ }^{\mathrm{TM}}-\mathrm{I}$ supplement, 2\% B27 and $25 \mu \mathrm{g} / \mathrm{ml}$ penicillin/streptomycin) without phenol red. Ara-C ( $2 \mu \mathrm{M}$; Sigma-Aldrich) was added 6-8 days after plating during the culture medium change, and cells were fed twice weekly thereafter. All the cells were grown at $37^{\circ} \mathrm{C}$ and in $5 \% \mathrm{CO}_{2}$.

Drugs and treatment. Hippocampal neurons were cultured with phenol red-free neurobasal cell culture medium throughout the whole culture period. Different concentrations of $17-\beta$-estradiol $(0,0.1$ and $1 \mathrm{ng} / \mathrm{ml})$ were added into the cell culture medium at 1, 3, 6 and 10 days in vitro (DIV), to maintain the neuron development and survival until the analysis. For the drug treatment study, the estrogen receptor antagonist ICI $182,780(100 \mathrm{nM})(20,21)$ and selective Kv4.2 and Kv4.3 channel blocker phrixotoxin2 (PaTx2) (100 nM) (22) were added to the culture medium with $17-\beta$-estradiol at the DIV1 for co-culturing.

Patch-clamp recordings data acquisition. Whole-cell recordings of pyramidal shaped neurons were recorded using a conventional patch-clamp technique with MultiClamp 700B amplifier (Axon Instruments, Foster City, CA, USA). Patch pipettes were pulled using a Sutter P-97 (Sutter Instrument, Novato, CA, USA) pipette puller. The bath solution contained $128 \mathrm{mM} \mathrm{NaCl}, 5 \mathrm{mM} \mathrm{KCl}, 25 \mathrm{mM}$ HEPES, $1 \mathrm{mM} \mathrm{MgCl}$, $30 \mathrm{mM}$ glucose and $2 \mathrm{mM} \mathrm{CaCl}_{2}$ (pH adjusted to 7.3 with $\mathrm{NaOH})$. Soft-glass recording pipettes were filled with an internal solution containing $125 \mathrm{mM}$ potassium gluconate, $10 \mathrm{mM} \mathrm{KCl}, 10 \mathrm{mM}$ HEPES, $5 \mathrm{mM}$ EGTA, $10 \mathrm{mM}$ Tris-phosphocreatine, $4 \mathrm{mM} \mathrm{MgATP}$ and $0.5 \mathrm{mM} \mathrm{Na}_{2} \mathrm{GTP}$ ( $\mathrm{pH}$ adjusted to 7.2-7.3 with $\mathrm{KOH}$ ). The pipette resistance was $3-5 \mathrm{M} \Omega$ subsequent to filling with internal solution. The cultured pyramidal shaped neurons selected for electrophysiological recording exhibited the same morphological characteristics. All the recordings were performed at room temperature.

In order to record the action potential, the membrane potential was held at $-70 \mathrm{mV}$ in the current clamp mode. A large depolarization shift was defined as a membrane potential up shift for $>10 \mathrm{mV}$, lasting for $\geq 300 \mathrm{msec}$. A bursting activity was defined by $\geq 5$ consecutive action potentials overlaying on the top of this large depolarization shift. When quantifying the percentage of neurons showing bursting activity, the criterion is $\geq 2$ bursts occurring during $10 \mathrm{~min}$ of recording, the same as our previous studies $(23,24)$.

$\mathrm{I}_{\mathrm{A}}$ recordings were obtained in the voltage clamp mode at $-100 \mathrm{mV}$. To record the fast transient outward potassium currents, the step command potentials were applied between -70 and $+40 \mathrm{mV}$, with $10-\mathrm{mV}$ steps and a width of $200 \mathrm{msec}$. In order to trigger the A-type potassium current, a prepulse $(-100 \mathrm{mV}, 200 \mathrm{msec})$ was applied to the cells immediately prior to the step commands (18). To isolate $\mathrm{I}_{\mathrm{A}}$, tetraethylamonium $(5 \mathrm{mM})$, tetrodotoxin $(1 \mu \mathrm{M})$ and $\mathrm{CdCl}_{2}(100 \mu \mathrm{M})$ were added to the extracellular solution to block the calcium channels, 
$A_{\text {a }}$

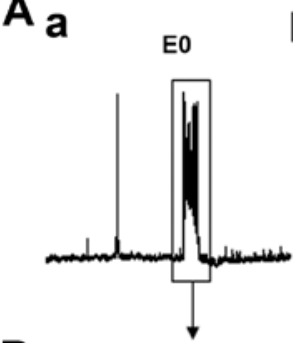

B a

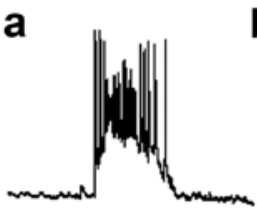

b1

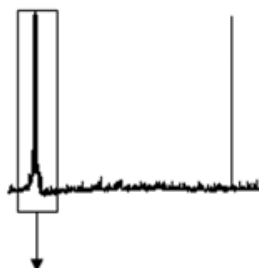

b1

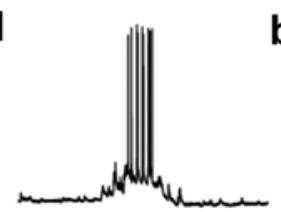

b2

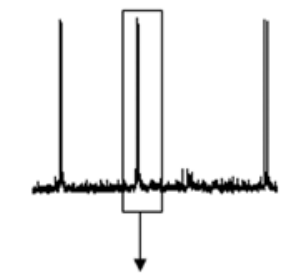

b2

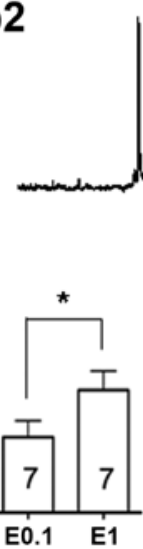

D

C

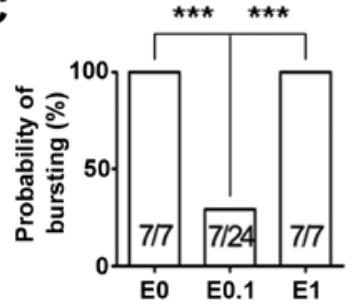

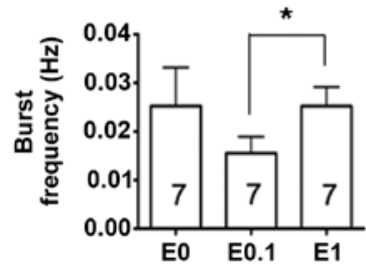

E

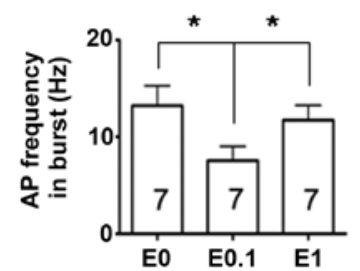

Figure 1. U-shape dose effects of 17- $\beta$-estradiol on neuronal burst activities in cultured hippocampal neurons. (A and B) Representative traces showing activities of neurons cultured with (a) 0 , (b) 0.1 and (c) $1 \mathrm{ng} / \mathrm{ml}$ (A) $17-\beta$-estradiol, and the traces in (B) are the enlarged images from (A) as indicated. (C-E) Bar histograms to show the group analysis of (C) the burst probability, (D) the averaged burst frequency and (E) the averaged inner burst action potential frequency in these three groups. Number of neurons analyzed were as indicated. The comparisons were tested by (C) $\chi^{2}$ test and (D and E) Student's t-test. ${ }^{*}<0.05$ and *** $\mathrm{P}<0.001$.

delayed rectifier potassium channels and voltage-gated sodium channels.

All the electrophysiological data were recorded using a MultiClamp 700B amplifier (Axon Instruments). Data acquisition and analysis were performed with pClamp 10.2 software (Axon Instruments).

Equation for curve fitting of the steady-state activation of $I_{A}$ currents. In the activation experiments, membrane potential was first held at $-100 \mathrm{mV}$. The total voltage-dependent potassium currents were evoked by a $200 \mathrm{msec}$ depolarization pulse from -70 to $+40 \mathrm{mV}$ in $10-\mathrm{mV}$ steps at 10 -sec intervals. The $\mathrm{I}_{\mathrm{A}}$ current value was the difference between the instant peak current and the steady-state current. Data were analyzed by calculation of the equation $\mathrm{G}=\mathrm{I} /\left(\mathrm{V}_{\mathrm{m}}-\mathrm{V}_{\text {rev }}\right)$, for the membrane potassium conductance. $\mathrm{V}_{\mathrm{m}}$ represents the membrane potential, whereas $\mathrm{V}_{\text {rev }}$ is the reversal potential of $\mathrm{K}^{+}(25)$. Subsequent to normalizing each current amplitude to the maximal current amplitude obtained from the depolarization to $+40 \mathrm{mV}$, the function $\mathrm{G} / \mathrm{G}_{\max }=1 /\left\{1+\exp \left[-\left(\mathrm{V}_{\mathrm{m}}-\mathrm{V}_{1 / 2}\right) / \mathrm{k}\right]\right\}$ was used to fit the data. From this equation, an activation curve of $I_{A}$ was obtained and the $\mathrm{V}_{1 / 2}$, the voltage at which the $\mathrm{I}_{\mathrm{A}}$ current was half-activated, was calculated.

Data analysis. All the electrophysiological results were analyzed using two-way analysis of variance (ANOVA) for comparisons between multiple groups, and post hoc analysis was performed with Fisher's least significant difference, $\chi^{2}$ test and the Student's t-test for direct comparison between the two groups. Data were all presented as mean \pm standard error of the mean and analyzed by SPSS 13.0 software (SPSS, Inc., Chicago, IL, USA). $\mathrm{P}<0.05$ was considered to indicate a statistically significant difference.

\section{Results}

$U$-shape dose-dependent effect of 17- $\beta$-estradiol on epileptiform bursting activities in cultured hippocampal neurons. In a previous study, we reported that phenol red, a weak estrogen receptor agonist, at the concentration of a supplement ingredient in the commercially available culture medium, has a suppressive action on epileptiform bursting activities in cultured neurons by activation of the estrogen receptors (13). Thus, in the present study, all the experiments were performed in phenol red-free medium cultured hippocampal neurons. In the phenol red-free neurobasal culture medium, DIV14 hippocampal neurons were cultured with alcohol (as the vehicle control for 17- $\beta$-estradiol) or 17- $\beta$-estradiol at doses of 0.1 and $1 \mathrm{ng} / \mathrm{ml}$, and were patch-clamp recorded in the current mode to record their firing properties (Fig. 1). Similar to the previous study (13), all the neurons $(7 / 7,100 \%)$ were showing epileptiform bursting activities while the neurons were cultured in phenol red-free medium with no added 17- $\beta$-estradiol $(0 \mathrm{ng} / \mathrm{ml})$. However, when the neurons were cultured in low dose $17-\beta$-estradiol $(0.1 \mathrm{ng} / \mathrm{ml})$-added medium, the percentage of neurons showing abnormal epileptiform bursting discharges $(7 / 24,29 \%)$ was significantly reduced $(\mathrm{P}<0.001)$ when compared to the vehicle control. By contrast, when the neurons were cultured in the high dose of $17-\beta$-estradiol $(1 \mathrm{ng} / \mathrm{ml})$, the percentage of neurons showing epileptiform bursting 


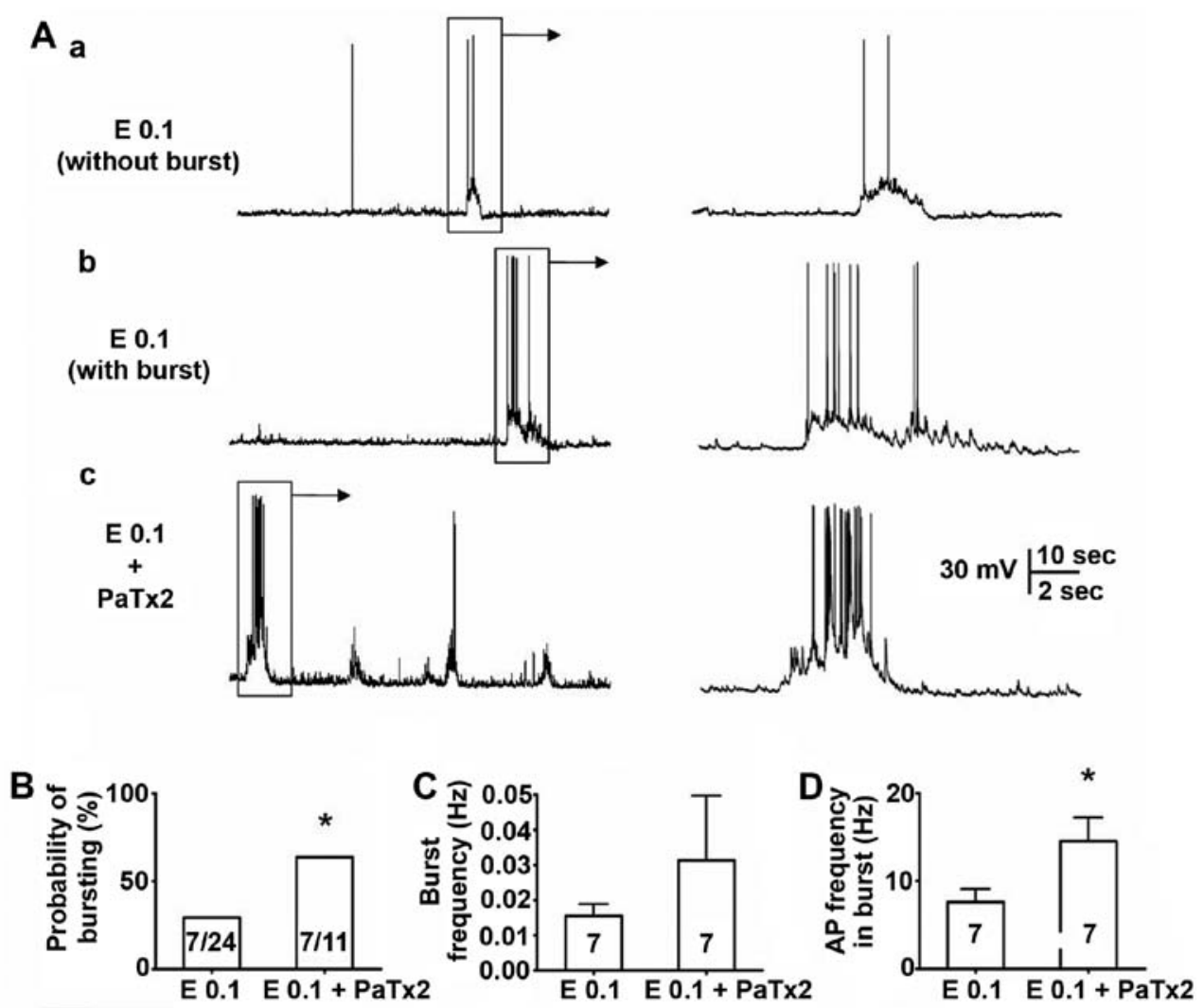

Figure 2. Inhibition of Kv4.2 channel with phrixotoxin2 (PaTx2) on the low dose 17- $\beta$-estradiol-induced suppressive effect on epileptiform burst activities. (A) Representative traces of the neuronal activities in the groups of neurons cultured in $0.1 \mathrm{ng} / \mathrm{ml}$ 17- $\beta$-estradiol (a and b) without or (c) with PaTx2 (100 nM). (B-D) The statistic analysis of (B) the burst probability, (C) the burst frequency and (D) the inner burst action potential frequency in these two groups. Number of neurons studied are as indicated. The comparisons were tested by the Student's t-test. " $\mathrm{P}<0.05$.

discharges $(7 / 7,100 \%)$ was not different to that of the vehicle control group, but significantly more than that of the low dose $17-\beta$-estradiol $(0.1 \mathrm{ng} / \mathrm{ml})$ group $(\mathrm{P}<0.001)$ (Fig. $1 \mathrm{~A}-\mathrm{C})$.

In addition, the burst frequency and the action potential frequency were analyzed within the burst among those bursting neurons recorded. The data revealed that when neurons were cultured with $0.1 \mathrm{ng} / \mathrm{ml} 17-\beta$-estradiol and had burst activities $(n=7)$, the burst frequency was not different to that of the neurons in the vehicle control group $(\mathrm{P}>0.05)$ (Fig. 1D); however, the action potential frequency within the burst was significantly lower $(\mathrm{P}<0.05)$ than that in the neurons in the vehicle control group (Fig. 1E). The burst frequency and the action potential frequency were further compared within the burst between the bursting neurons from neurons cultured with either low $(0.1 \mathrm{ng} / \mathrm{ml})$ or high $(1 \mathrm{ng} / \mathrm{ml}) 17-\beta$-estradiol medium. The results showed that the burst frequency and the action potential frequency within the burst among those bursting neurons were significantly lower $(\mathrm{P}<0.05)$ in neurons treated with $0.1 \mathrm{ng} / \mathrm{ml} 17-\beta$-estradiol compared with those in

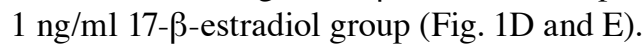

These results indicate that the effect of $17-\beta$-estradiol on the epileptiform bursting discharges is U-shape like, and $17-\beta$-estradiol, in a certain low dose range, has a suppressing effect, similar to the effect that we previously reported (13).

Effect of PaTx2 on epileptiform bursting activities. Previous studies have shown that $17-\beta$-estradiol has a modulation effect on the transient (mediated by Kv4.2) and sustained outward potassium current (major mediated by Kv2 family) (18), and $\mathrm{Kv} 4.2$ is associated with the seizure occurrence (12). Thus, whether the suppressive effect of the low dose $(0.1 \mathrm{ng} / \mathrm{ml})$ $17-\beta$-estradiol on spontaneous epileptiform bursting activities in cultured neurons is due to modulation of the transient outward potassium current mediated by $\mathrm{Kv} 4.2$ was investigated using the selective Kv4.2/Kv4.3 channel blocker PaTx2. After 14 days co-culturing in low dose $17-\beta$-estradiol $(0.1 \mathrm{ng} / \mathrm{ml})$ with PaTx2 (100 nM), neurons at DIV14 were patch-clamp studied of their spontaneous activities (Fig. 2). As expected, PaTx2 significantly blocked the low dose 17- $\beta$-estradiol-induced suppressive effect on the epileptiform activities (Fig. 2A). The percentage of neurons showing epileptiform activity in the PaTx2-treated group was $64 \%$ (7 in 11), which was significantly higher than those cultured with low dose $17-\beta$-estradiol alone (29\%, 7 in 24 ; $\mathrm{P}<0.05)$ (Fig. 2B). In addition, PaTx 2 also significantly inhibited low dose $17-\beta$-estradiol-induced reduction of the average action potential frequency within the burst $(\mathrm{P}<0.05)$ (Fig. 2D). It should be noted that we did not compare the effect of PaTx 2 and high dose 17- $\beta$-estradiol on bursting activity in this study, but aim to do so in the future.

This result indicates that the potassium channel $\mathrm{Kv} 4.2$ is possibly involved in the low dose of $17-\beta$-estradiol-induced suppressive effect on epileptiform activities.

Dose-dependent effect of 17- $\beta$-estradiol on fast transient outward potassium currents in cultured hippocampal neurons. In order to study the mechanism of how $17-\beta$-estradiol 


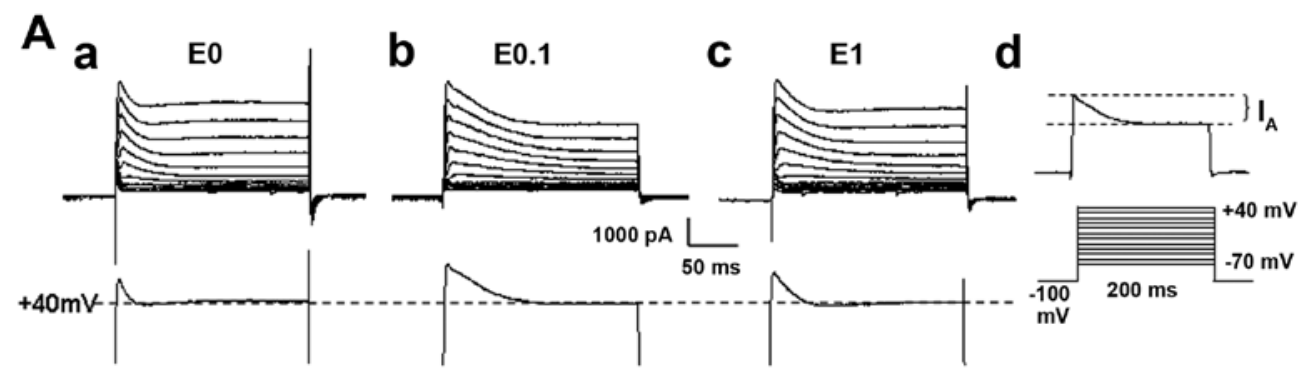

B

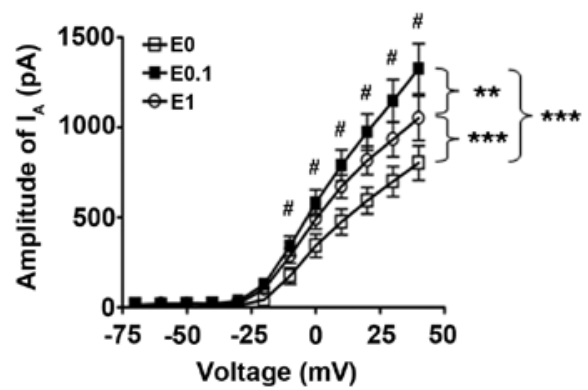

C

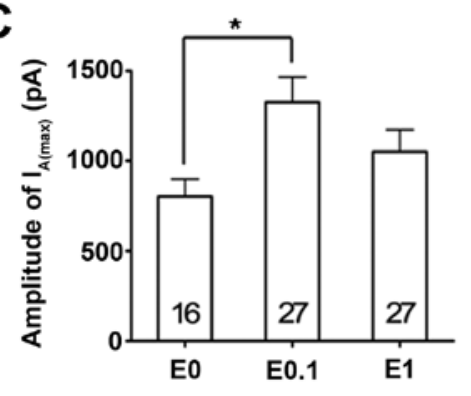

Figure 3. Dose-dependent effects of 17- $\beta$-estradiol on potassium $\mathrm{I}_{\mathrm{A}}$ currents. (A) Representative original traces of the (a-c) steady-step voltage change-evoked potassium channel currents, and (d) the schematic diagram of $\mathrm{I}_{\mathrm{A}}$ measurement and the recording voltage ladder. (a-c) The single traces below are the $\mathrm{I}_{\mathrm{A}}$ currents obtained at a membrane voltage of $+40 \mathrm{mV}$. (B) The $\mathrm{I}-\mathrm{V}$ curves of the $\mathrm{I}_{\mathrm{A}}$ change at the voltage step from -70 to $+40 \mathrm{mV}$ in the culture groups treated with 17- $\beta$-estradiol at $0(\mathrm{n}=16), 0.1(\mathrm{n}=27)$ and $1(\mathrm{n}=27) \mathrm{ng} / \mathrm{ml} .{ }^{* *} \mathrm{P}<0.01$ and ${ }^{* * *} \mathrm{P}<0.001$, respectively, as tested by two-way analysis of variance, and ${ }^{\#} \mathrm{P}<0.05$ represented the comparison between E0 and E0.1 groups by post-hoc analyses. $(\mathrm{C})$ Comparison of the amplitude of maximal amplitude of the $\mathrm{I}_{\mathrm{A}}\left(\mathrm{I}_{\mathrm{A}(\max )}\right)$ tested at $+40 \mathrm{mV}$. $\mathrm{P}<0.05$ by the Student's t-test.

modulates epileptiform activity of hippocampal neurons through the potassium Kv4.2 channel, the fast transient outward potassium currents $\left(\mathrm{I}_{\mathrm{A}}\right)$, which are mostly mediated by Kv4.2 channel, were further tested by the patch-clamp technique in different $17-\beta$-estradiol-treated neuron groups. Similar to the effect of $17-\beta$-estradiol on epileptiform bursting activities in cultured hippocampal neurons, a dose-related 'bell' shape-like effect of 17- $\beta$-estradiol on fast transient outward potassium currents was identified (Fig. 3). Statistical analysis of the $\mathrm{I}-\mathrm{V}$ curves of the $\mathrm{I}_{\mathrm{A}}$ revealed that low $(0.1 \mathrm{ng} / \mathrm{ml}, \mathrm{n}=27)$ and high dose $(1 \mathrm{ng} / \mathrm{ml}, \mathrm{n}=27)$ 17- $\beta$-estradiol significantly enhanced $\mathrm{I}_{\mathrm{A}}$ compared to the $17-\beta$-estradiol-free vehicle control group $(\mathrm{P}<0.001$, two-way ANOVA) (Fig. 3A and B). However, further statistical analyses between the low and high dose of the $17-\beta$-estradiol-treated groups data demonstrated that the low dose of $17-\beta$-estradiol $(0.1 \mathrm{ng} / \mathrm{ml})$ had the largest enhancement effect on the $I_{A}$, while further increasing the $17-\beta$-estradiol concentration to a higher dose $(1 \mathrm{ng} / \mathrm{ml})$ caused a reduction, although this remained higher compared to the vehicle control group and did not further enhance on the $\mathrm{I}_{\mathrm{A}}(\mathrm{P}<0.01$, two-way ANOVA) (Fig. 3A and B). However, the post-hoc analysis showed that only the low dose of $17-\beta$-estradiol had significant enhancement on the $\mathrm{I}_{\mathrm{A}}(\mathrm{P}<0.05)$ (Fig. 3B).

The differences of the maximal amplitude of the $\mathrm{I}_{\mathrm{A}}\left(\mathrm{I}_{\mathrm{A}(\max )}\right)$, which was evoked while the current command was at $+40 \mathrm{mV}$, was further studied among those groups. The data showed that the $\mathrm{I}_{\mathrm{A}(\max )}$ was $1,325 \pm 139 \mathrm{pA}(\mathrm{n}=27)$ in the low dose of $17-\beta$-estradiol $(0.1 \mathrm{ng} / \mathrm{ml})$ group, which was significantly higher than that of the $\mathrm{I}_{\mathrm{A}(\max )}(802 \pm 96 \mathrm{pA}, \mathrm{n}=16, \mathrm{P}<0.05)$ in the vehicle control group (17- $\beta$-estradiol at $0 \mathrm{ng} / \mathrm{ml})$. By contrast, the $\mathrm{I}_{\mathrm{A}(\max )}$ in the high dose of $17-\beta$-estradiol $(1 \mathrm{ng} / \mathrm{ml})$ group was $1,050 \pm 122 \mathrm{pA}(\mathrm{n}=27)$, which was not different to that of the neurons tested in the vehicle control group $(\mathrm{P}>0.05)$.
These data indicate that $\mathrm{I}_{\mathrm{A}}$ is capable of being modulated by the long-term estrogen receptor stimulation at a low dose of $17-\beta$-estradiol, which enhanced the $\mathrm{I}_{\mathrm{A}}$ amplitude.

Effect of ICI 182,780 on the suppressive action of low dose $17-\beta$-estradiol on the $I_{A}$ currents in cultured hippocampal neurons. As 17- $\beta$-estradiol treatment at the low and high concentration had a modulation effect on the potassium $\mathrm{I}_{\mathrm{A}}$, whether the enhancement of the low dose of 17- $\beta$-estradiol on the potassium $\mathrm{I}_{\mathrm{A}}$ is mediated by the estrogen receptors was further analyzed using ICI 182,780, an estrogen receptor antagonist.

When neurons were co-cultured with $17-\beta$-estradiol at 0.1 or $1 \mathrm{ng} / \mathrm{ml}$ and with ICI $182,780(100 \mathrm{nM})$, the statistical analysis of the I-V curves showed that antagonism of the estrogen receptors with ICI 182,780 significantly suppressed the low ( $\mathrm{P}<0.05, \mathrm{n}=34$, two-way ANOVA) (Fig. 4A) and high $(\mathrm{P}<0.01, \mathrm{n}=22$, two-way ANOVA) (Fig. 4B) dose $17-\beta$-estradiol-induced potassium $\mathrm{I}_{\mathrm{A}}$ enhancement in comparison with the 17- $\beta$-estradiol treatment alone groups $(n=27$ and $\mathrm{n}=27$, respectively) (Fig. 4B and C).

In addition to suppression of the $I_{A}$ amplitude, the antagonism of the estrogen receptor with ICI 182,780 also significantly left-shifted the steady-state activation curve of the $\mathrm{I}_{\mathrm{A}}$ currents, which represents the channel-opening properties of the Kv4.2 potassium channels under the low dose $17-\beta$-estradiol $(0.1 \mathrm{ng} / \mathrm{ml})$ culture condition. The $\mathrm{I}_{\mathrm{A}}$ current half-activation potential $\left(\mathrm{V}_{1 / 2}\right)$ underwent a significant depolarization shift increase from $-7.48 \pm 1.6 \mathrm{mV}$ $(\mathrm{n}=27)$ to $0.00 \pm 1.8 \mathrm{mV}(\mathrm{n}=34)(\mathrm{P}<0.05)$ in the neuron groups with $17-\beta$-estradiol $(0.1 \mathrm{ng} / \mathrm{ml})$ alone or co-treatment with ICI 182,780, respectively (Fig. 5).

These data indicate that $\mathrm{I}_{\mathrm{A}}$ is capable of being modulated by the long-term estrogen receptor stimulation at the low dose of 
A $\quad$ E0.1

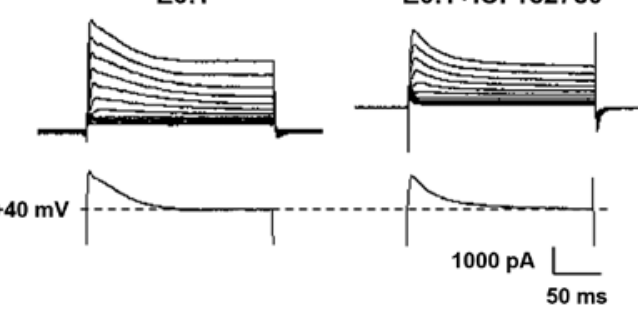

B

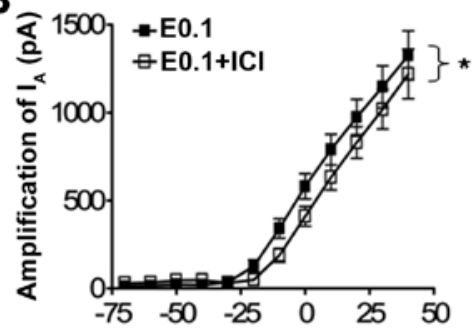

C

E1

E1+ICI 182780
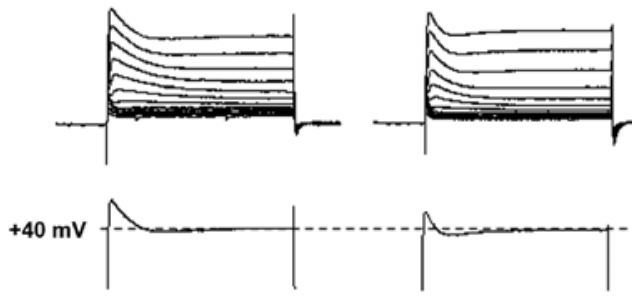

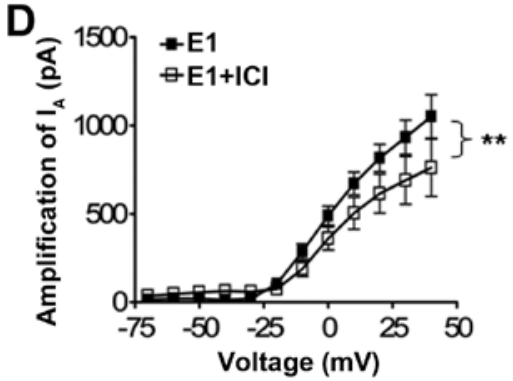

Figure 4. Estrogen receptor antagonist ICI 182,780 inhibits the 17- $\beta$-estradiol-induced enhancement of potassium $\mathrm{I}_{\mathrm{A}}$ current. (A and C) Representative original traces of potassium currents evoked by the steady-step voltage change from -70 to $+40 \mathrm{mV}$ (upper traces), and the single-traces at voltage of $+40 \mathrm{mV}$ (bottom traces). (B and D) The I-V curves of the $\mathrm{I}_{\mathrm{A}}$ change at the voltage step from -70 to $+40 \mathrm{mV}$ in the co-culture groups treated with ICI 182,780 (100 $\mathrm{nM}$ ) and 17- $\beta$-estradiol at either $0.1(\mathrm{n}=34)$ or $1(\mathrm{n}=22) \mathrm{ng} / \mathrm{ml}$. ${ }^{*} \mathrm{P}<0.05$ and ${ }^{* * *} \mathrm{P}<0.01$, respectively, as compared with $17-\beta$-estradiol at $0.1(\mathrm{n}=27)$ and $1(\mathrm{n}=27) \mathrm{ng} / \mathrm{ml}$ alone by two-way analysis of variance.
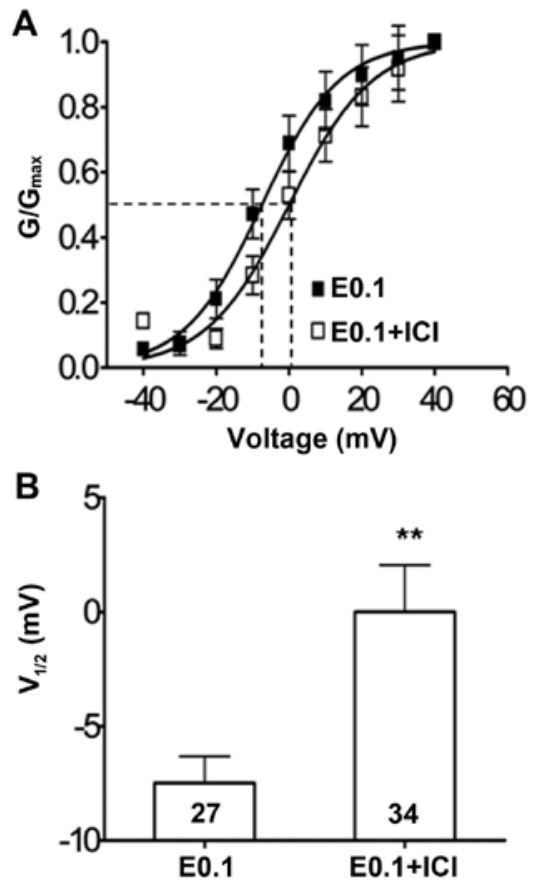

Figure 5. ICI 182,780 alters the $\mathrm{I}_{\mathrm{A}}$ steady-state activation curve to a depolarizing level. (A) $\mathrm{I}_{\mathrm{A}}$ steady-state activation curves obtained by plotting the normalized conductance as a function of command potential obtained from neuron groups treated with low dose $17-\beta$-estradiol $(0.1 \mathrm{ng} / \mathrm{ml})(\mathrm{n}=27)$ and with or without estrogen receptor antagonist ICI 182,780 $(100 \mathrm{nM})(\mathrm{n}=34)$ Data points were fitted by the Boltzmann function. ICI 182,780 significantly right shifted the steady-state activation curves ( $\mathrm{G}$ represents conductance). (B) Bar histogram showing ICI 182,780 significantly depolarizing shifted the $\mathrm{V}_{1 / 2}{ }^{* *} \mathrm{P}<0.01$.

17- $\beta$-estradiol, which enhanced the potassium Kv4.2 channel activities by enhancing $\mathrm{I}_{\mathrm{A}}$ amplitude and channel-opening probability.

\section{Discussion}

The present experimental results demonstrated that $17-\beta$-estradiol, the biologically active estrogen, had a regulative action on abnormal epileptiform bursting activities in cultured hippocampal neurons in a U-shape dose-dependent manner: Extremely low or high concentrations of $17-\beta$-estradiol enhance, but low concentrations of $17-\beta$-estradiol suppress, the epileptiform bursting activities in the cultured hippocampal neurons. This suppressive effect of $17-\beta$-estradiol on the epileptiform bursting activities is possibly due to, at least in part, the modulation of the potassium Kv4.2 channel activities by activation of estrogen receptors.

In the present study, the dose effect of estrogen receptor stimulation on the epileptiform bursting activities in cultured hippocampal neurons was examined. Estrogen has been reported to have a differential effect on neuronal function. $17-\beta$-estradiol has been reported to increase the excitability of gonadotrophin-releasing hormone neurons (26), medial vestibular nucleus neurons in brain stem (27) and hippocampal neurons (28). By contrast, estrogen receptor activation has also been reported to decrease neuronal excitability by indirectly changing the local neurotransmitter release (29), particularly by changing the interaction with GABAergic neurons $(30,31)$. Our previous study also indicated that on the same hippocampal neuron, a weak estrogen receptor agonist, phenol red, could have a U-shape-like activation-inhibition-activation effect on the epileptiform bursting activities: Low and high concentrations of phenol red all induced the epileptiform bursting activities in the cultured hippocampal neurons, while the middle concentration $(\sim 28 \mu \mathrm{M})$ of phenol red suppressed this activity (13). Similar to the previous reports, a large proportion of the neurons cultured in the estrogen-free medium had epileptiform bursting activities in the present study, and 
the low dose $17-\beta$-estradiol $(\sim 0.1 \mathrm{ng} / \mathrm{ml})$ had a suppressive effect, whereas the high dose $17-\beta$-estradiol $(1 \mathrm{ng} / \mathrm{ml})$ had a promoting effect on the neuronal excitability change, forming a U-shape-like dose-dependent action on the neuronal excitation change. This effect may explain the differential action of the estrogen receptor stimulation caused increase or decrease of neuronal excitability in various studies (28-31).

Neuron excitability is determined by various factors; one of them is the open properties of the potassium channels. Voltage-gated potassium (Kv) channels are important for maintaining the membrane potentials, action potential shape, firing adaptation and neuronal excitability in neurons (4). The Kv channels-mediated current contains the transient and the sustained current $(5,6)$. Previous studies have demonstrated that the transient outward $\mathrm{I}_{\mathrm{A}}$ has an important role in controlling the membrane excitability and that it contributes to remodeling neuronal excitation under pathological conditions $(7,8)$. In the present study, the modulatory effect of low dose $17-\beta$-estradiol on the epileptiform bursting probability was blocked by the selective Kv4.2 and 4.3 potassium channel blocker PaTx2, indicating the involvement of the Kv4.2 and 4.3 potassium channels. The effect of $17-\beta$-estradiol on the voltage-gated fast transient outward potassium current was further examined to improve the understanding of how $17-\beta$-estradiol affects neuronal excitability and the probability of the epileptiform bursting activity occurrence. Using whole cell clamp recordings, the results demonstrated that $17-\beta$-estradiol had a dose-associated modulatory effect on the voltage-gated fast transient outward $\mathrm{I}_{\mathrm{A}}$ curve and on the maximal current measured when the membrane potential was transiently increased to $+40 \mathrm{mV}$. The low and high dose of $17-\beta$-estradiol significantly increased the amplitude of the $I_{A}$. Notably, the low concentration of $17-\beta$-estradiol at $0.1 \mathrm{ng} / \mathrm{ml}$ had the strongest effect on the increase of the amplitude of $I_{A}$, which is also significantly more than that of $17-\beta$-estradiol at $1 \mathrm{ng} / \mathrm{ml}$, showing a bell-shape concentration-dependent manner on the facilitation of the voltage-gated fast transient outward $\mathrm{I}_{\mathrm{A}}$. These results are consistent with the finding that a low dose of $17-\beta$-estradiol only had a significant suppressive effect on the epileptiform bursting activities, which showed a U-shape concentration-dependent inhibitory action. As the transient outward $\mathrm{I}_{\mathrm{A}}$ has an important role in controlling the membrane excitability and remodeling neuronal excitation under pathological conditions $(7,8)$, the present results indicate that activation of estrogen receptors on modulating neuronal epileptiform bursting activities may, through the mechanism of modulating the transient outward $\mathrm{I}_{\mathrm{A}}$, alter the cell excitability.

Estrogen is one of the main hormones of female mammals (however, it also exists in males), which has a complex and wide physiological and pathophysiological effect, such as promoting cell proliferation (32-34) as well as modulating the neuronal excitability $(3,27,28,35-37)$, and the effect of $17-\beta$-estradiol, an estrogen receptor agonist, observed in the present study is possibly due to activation of the estrogen receptors, as the facilitation effect on the transient outward $\mathrm{I}_{\mathrm{A}}$ in cultured hippocampal neurons was inhibited by the estrogen receptor antagonist ICI 182,780 (20,21) (Fig. 4). However, the results from the present study could not distinguish which subtype of the estrogen receptors was mediating this function, as ICI 182,780 is neither an $\alpha$ - nor $\beta$-estrogen receptor subtype-selective antagonist. The reason why $17-\beta$-estradiol has an effect on the transient outward $\mathrm{I}_{\mathrm{A}}$, as well as on the neuronal epileptiform bursting activities, in a bell-shape (U-shape) mannor remains unknown. We hypothesize that the estrogen receptor subtype may have a major role in differentially modulating neuronal excitability with regards to the firing properties. The involvement of either the $\alpha$ - or $\beta$-subtype estrogen receptors in mediating this $17-\beta$-estradiol action requires further study using selective antagonists for $\alpha$ and $\beta$-estrogen receptor subtypes in the future.

Potassium channels are important for post-excitatory membrane repolarization and sustain different components of hyperpolarizing after-potentials in intrinsically bursting cells. The inhibition of $\mathrm{K}^{+}$currents interferes with repolarization and hyperpolarization, and increases hyperexcitability and bursting activity (38). In the Kv channel family, Kv4 is the major subtype mediating the transient outward $\mathrm{I}_{\mathrm{A}}(9,10)$. In the hippocampus, dendrites of CA1 pyramidal neurons contain a high density of transient A-type potassium channels, and A-type $\mathrm{K}^{+}$channels are crucial modulators of information processing and synaptic plasticity in the dendrites $(9,10)$. A reduction in A-type $\mathrm{K}^{+}$ channel activity promotes burst firing in a location-dependent manner (39). The changes in the potassium currents alter shape and repetitive frequency of the action potentials. The presence of the rapidly inactivating A-type channels maintains the firing rate at a low frequency and broadens the action potentials when neurons fire repetitively (40). These two changes diminish the firing rate in the neurons. This indicates that the A-type potassium current is a type of inhibitory effect in the normal physiological condition in the hippocampus. In the present study, the neurons with a high probability to have epileptiform bursting, such as neurons in the estrogen-free culture group, also have a high firing frequency of the action potentials in the burst, and the lowest $\mathrm{I}_{\mathrm{A}}$. Increasing the $\mathrm{I}_{\mathrm{A}}$ by low dose of $17-\beta$-estradiol significantly reduced the action potential firing frequency along with the inhibited epileptiform bursting probability and the suppressed bursting frequency. This result indicates that there is a negative correlation between the transient outward potassium current and the epileptiform bursting properties, including bursting frequency and bursting action potential frequency. This is consistent with the previous findings that in pilocarpine seizure rats, the increased dendritic excitability and the epileptic seizure generation is possibly due to the decreased A-type potassium channels activities (12).

The results from the present study suggest that estrogen receptor activation is important for modulating the neuronal cell excitability and maintaining the normal neuronal physiological conditions, including the firing properties of the neurons, by modulating the potassium channel activities. As estrogen receptor stimulation could either enhance the neuronal excitability by directly modulating the intrinsic properties $(28,36)$ or, in the other direction, decrease the neuronal excitability by indirectly changing the local neurotransmitter release $(29,35)$ particularly by interaction with GABAergic neurons $(29,30)$, the present experimental results may indicate that, at least in part, the activation of estrogen receptors, with a certain range of the estradiol concentration, in modulating the neuronal bursting activities in the cultured hippocampal neurons is possibly directly modulating the potassium channel, particularly the Kv4.2 channel, properties by enhancing the channel current and channel-opening probability. 
In conclusion, the present study identified that the estrogen level at a certain physiological concentration is important in maintaining the potassium channel activities and in turn influences the neuronal excitability to the extent of the epileptiform activities. Thus, the present results indicate that reduced activation of the transient outward potassium current by a high estrogen level may be one of the causes for triggering catamenial epilepsy, and provides us with a potential therapeutical target to intervene with catamenial epilepsy in the future.

\section{Acknowledgements}

The present study was supported by grants from the Nature Science Foundation of China (nos. 31129003, 81171224, 31271188 and 81301108) and the Science and Technology Commission of Shanghai Municipality (nos. 13DJ1400302 and 13ZR1406500) to YW., X.L. and XW.

\section{References}

1. Newmark ME and Penry JK: Catamenial epilepsy: A review. Epilepsia 21: 281-300, 1980.

2. Laidlaw J. Catamenial epilepsy. Lancet 271: 1235-1237, 1956.

3 . Velísková J: The role of estrogens in seizures and epilepsy: The bad guys or the good guys? Neuroscience 138: 837-844, 2006.

4. Takeda M, Tsuboi Y, Kitagawa J, Nakagawa K, Iwata K and Matsumoto S: Potassium channels as a potential therapeutic target for trigeminal neuropathic and inflammatory pain. Mol Pain 7: 5, 2011.

5. Iverson LE, Tanouye MA, Lester HA, Davidson N and Rudy B: A-type potassium channels expressed from Shaker locus cDNA. Proc Natl Acad Sci USA 85: 5723-5727, 1988.

6. Solc CK, Zagotta WN and Aldrich RW: Single-channel and genetic analyses reveal two distinct A-type potassium channels in Drosophila. Science 236: 1094-1098, 1987.

7. Sonner PM and Stern JE: Functional role of A-type potassium currents in rat presympathetic PVN neurones. J Physiol 582: 1219-1238, 2007.

8. Sonner PM, Filosa JA and Stern JE: Diminished A-type potassium current and altered firing properties in presympathetic PVN neurones in renovascular hypertensive rats. J Physiol 586: 1605-1622, 2008.

9. Covarrubias M, Wei AA and Salkoff L: Shaker, Shal, Shab, and Shaw express independent $\mathrm{K}^{+}$current systems. Neuron 7: 763-773, 1991.

10. Serôdio P, Vega-Saenz de Miera E and Rudy B: Cloning of a novel component of A-type $\mathrm{K}^{+}$channels operating at subthreshold potentials with unique expression in heart and brain. J Neurophysiol 75: 2174-2179, 1996.

11. Hoffman DA, Magee JC, Colbert CM and Johnston D: $\mathrm{K}^{+}$channel regulation of signal propagation in dendrites of hippocampal pyramidal neurons. Nature 387: 869-875, 1997.

12. Bernard C, Anderson A, Becker A, Poolos NP, Beck H and Johnston D: Acquired dendritic channelopathy in temporal lobe epilepsy. Science 305: 532-535, 2004.

13. Liu X, Chen B, Chen L, Ren WT, Liu J, Wang G, Fan W, Wang X and Wang Y: U-shape suppressive effect of phenol red on the epileptiform burst activity via activation of estrogen receptors in primary hippocampal culture. PLoS One 8: e60189, 2013.

14. Hoffman GE, Moore N, Fiskum G and Murphy AZ: Ovarian steroid modulation of seizure severity and hippocampal cell death after kainic acid treatment. Exp Neurol 182: 124-134, 2003.

15. Carroll JC, Rosario ER and Pike CJ: Progesterone blocks estrogen neuroprotection from kainate in middle-aged female rats. Neurosci Lett 445: 229-232, 2008.

16. Fatehi M, Kombian SB and Saleh TM: 17beta-estradiol inhibits outward potassium currents recorded in rat parabrachial nucleus cells in vitro. Neuroscience 135: 1075-1086, 2005.

17. Bosch MA, Kelly MJ and Rønnekleiv OK: Distribution, neuronal colocalization, and 17beta-E2 modulation of small conductance calcium-activated $\mathrm{K}(+)$ channel (SK3) mRNA in the guinea pig brain. Endocrinology 143: 1097-1107, 2002.
18. Farkas I, Varju P and Liposits Z: Estrogen modulates potassium currents and expression of the Kv4.2 subunit in GT1-7 cells. Neurochem Int 50: 619-627, 2007.

19. Chen B, Jiang M, Zhou M, Chen L, Liu X, Wang X and Wang Y: Both NMDA and non-NMDA receptors mediate glutamate stimulation induced cofilin rod formation in cultured hippocampal neurons. Brain Res 1486: 1-13, 2012.

20. Howell A, Osborne CK, Morris C and Wakeling AE: ICI 182,780 (Faslodex): Development of a novel, 'pure' antiestrogen. Cancer 89: 817-825, 2000.

21. Wong JK, Le HH, Zsarnovszky A and Belcher SM: Estrogens and ICI182,780 (Faslodex) modulate mitosis and cell death in immature cerebellar neurons via rapid activation of $\mathrm{p} 44 / \mathrm{p} 42$ mitogen-activated protein kinase. J Neurosci 23: 4984-4995, 2003.

22. Bosmans F, Rash L, Zhu S, Diochot S, Lazdunski M, Escoubas P and Tytgat J: Four novel tarantula toxins as selective modulators of voltage-gated sodium channel subtypes. Mol Pharmacol 69: 419-429, 2006.

23. Qi J, Wang Y, Jiang M, Warren P and Chen G: Cyclothiazide induces robust epileptiform activity in rat hippocampal neurons both in vitro and in vivo. J Physiol 571: 605-618, 2006.

24. Wang Y, Qi JS, Kong S, Sun Y, Fan J, Jiang M and Chen G: BDNF-TrkB signaling pathway mediates the induction of epileptiform activity induced by a convulsant drug cyclothiazide. Neuropharmacology 57: 49-59, 2009.

25. Zhuang JL, Wang CY, Zhou MH, Duan KZ and Mei YA: TGF- $\beta 1$ enhances Kv2.1 potassium channel protein expression and promotes maturation of cerebellar granule neurons. J Cell Physiol 227: 297-307, 2012.

26. Rønnekleiv OK, Bosch MA and Zhang C: $17 \beta$-oestradiol regulation of gonadotrophin-releasing hormone neuronal excitability. J Neuroendocrinol 24: 122-130, 2012.

27. Grassi S, Frondaroli A, Scarduzio M, Dutia MB, Dieni C and Pettorossi VE: Effects of 17beta-estradiol on glutamate synaptic transmission and neuronal excitability in the rat medial vestibular nuclei. Neuroscience 165: 1100-1114, 2010.

28. Zadran S, Qin Q, Bi X, Zadran H, Kim Y, Foy MR, Thompson R and Baudry $\mathrm{M}$ : 17-Beta-estradiol increases neuronal excitability through MAP kinase-induced calpain activation. Proc Natl Acad Sci USA 106: 21936-21941, 2009.

29. Saleh TM, Connell BJ, McQuaid T and Cribb AE: Estrogen-induced neurochemical and electrophysiological changes in the parabrachial nucleus of the male rat. Brain Res 990: 58-65, 2003.

30. Blurton-Jones M and Tuszynski MH: Estrogen receptor-beta colocalizes extensively with parvalbumin-labeled inhibitory neurons in the cortex, amygdala, basal forebrain, and hippocampal formation of intact and ovariectomized adult rats. J Comp Neurol 452: 276-287, 2002.

31. Zhou J,Pfaff DW and Chen G: Sex differences in estrogenic regulation of neuronal activity in neonatal cultures of ventromedial nucleus of the hypothalamus. Proc Natl Acad Sci USA 102: 14907-14912, 2005.

32. Frankfurt M, Gould E, Woolley CS and McEwen BS: Gonadal steroids modify dendritic spine density in ventromedial hypothalamic neurons: A Golgi study in the adult rat. Neuroendocrinology 51: 530-535, 1990.

33. Woolley CS and McEwen BS: Estradiol mediates fluctuation in hippocampal synapse density during the estrous cycle in the adult rat. J Neurosci 12: 2549-2554, 1992.

34. Calizo LH and Flanagan-Cato LM: Estrogen selectively regulates spine density within the dendritic arbor of rat ventromedial hypothalamic neurons. J Neurosci 20: 1589-1596, 2000.

35. Velísková J and Velísek L: Beta-estradiol increases dentate gyrus inhibition in female rats via augmentation of hilar neuropeptide Y. J Neurosci 27: 6054-6063, 2007.

36. Edwards HE, Burnham WM, Mendonca A, Bowlby DA and MacLusky NJ: Steroid hormones affect limbic afterdischarge thresholds and kindling rates in adult female rats. Brain Res 838: 136-150, 1999.

37. Herzog AG: Catamenial epilepsy: Definition, prevalence pathophysiology and treatment. Seizure 17: 151-159, 2008.

38. Ptacek LJ and Fu YH: Channelopathies: Episodic disorders of the nervous system. Epilepsia 42 (Suppl 5): 35-43, 2001.

39. Johnston D, Hoffman DA, Magee JC, Poolos NP, Watanabe S, Colbert CM and Migliore M: Dendritic potassium channels in hippocampal pyramidal neurons. J Physiol 525: 75-81, 2000.

40. Connor JA and Stevens CF: Prediction of repetitive firing behaviour from voltage clamp data on an isolated neurone soma. J Physiol 213: 31-53, 1971. 